\title{
List A Trials 1-5
}

National Cancer Institute

\section{Source}

National Cancer Institute. List A Trials 1-5. NCI Thesaurus. Code C120390.

The first part of the California Verbal Learning Test for Children. For the first five trials, a 15-item list, List $A$, is read to the child and he or she is asked to recall words from the list after each presentation. 